\title{
Modelado Matemático de un Controlador PID de Ganancia Variable dependiente del Punto de Consigna en la Salida de un PID Convencional
}

\section{Mathematical Modeling of a Variable Gain PID Controller, Dependent of the set point in the output of a Conventional PID}

\author{
GARCÍA-CORTÉS, Julio Zenón ${ }^{1} \dagger^{*}$, CARRILLO-GARCÍA, Francisco Javier ${ }^{1}$, TAMAYO-LOEZA, \\ Erick del Jesus ${ }^{2}$ y MAY-CEN, Ivan de Jesus ${ }^{2}$ \\ ${ }^{I}$ Departamento de Ingeniería Electromecánica, Instituto Tecnológico Superior Zacatecas Norte, Río Grande, Zacatecas, \\ México \\ ${ }^{2}$ Departamento de Ingeniería Electromecánica, Instituto Tecnológico Superior Progreso, Progreso, Yucatán, México
}

ID $1^{\text {er }}$ Autor: Julio Zenón, García-Cortés / ORC ID: 0000-0003-2321-619X, Researcher ID Thomson: T-3638-2018, CVU CONACYT ID: 294610

ID $1^{\mathrm{er}}$ Autor: Francisco Javier, Carrillo-García / ORC ID: 0000-0002-6071-0990, Researcher ID Thomson: T-4599-2018 ID $2^{\text {do }}$ Autor: Erick del Jesus, Tamayo-Loeza / ORC ID: 0000-0003-2763-0742, Researcher ID Thomson: T-4605-2018, CVU CONACYT ID: 298427

ID $3^{\text {er }}$ Autor: Ivan de Jesus, May-Cen / ORC ID: 0000-0003-4147-212X, Researcher ID Thomson: T-4598-2018, CVU CONACYT ID: 226576

DOI: $10.35429 /$ JTO.2019.12.3.1.6

Recibido 22 de Octubre, 2019, Aceptado, 24 de Noviembre, 2019

\section{Resumen}

Se modificó la estructura de control PID mostrada en la literatura por K. Ogata. Se hace la propuesta de una estructura de control PID de ganancia variable, implementada mediante electrónica analógica. Se obtuvo un modelo matemático utilizando métodos convencionales. Se comprobó la validez del modelo obtenido comparando contra la respuesta del modelo planteado por K. Ogata. Se concluyó que la estructura propuesta es estable.

Modelado, Sistemas de Control no lineales, PID

\begin{abstract}
A PID control structure was modified proposed by K. Ogata. It is proposed a structure Nonlinear PID control, implemented using analogical electronics. A mathematical model was obtained using conventional methods. It was proved the validity of the model obtained comparing against the response of the model proposed by K. Ogata. It was concluded that the proposed structure is stable.
\end{abstract}

Modeling, Nonlinear control systems, PID

Citación: GARCÍA-CORTÉS, Julio Zenón, CARRILLO-GARCÍA, Francisco Javier, TAMAYO-LOEZA, Erick del Jesus y MAY-CEN, Ivan de Jesus. Modelado Matemático de un Controlador PID de Ganancia Variable dependiente del Punto de Consigna en la Salida de un PID Convencional. Revista de Operaciones Tecnológicas. 2019 3-12: 1-6

\footnotetext{
* Correspondencia del Autor (Correo electrónico: profejulioz@ hotmail.com)

$\dagger$ Investigador contribuyendo como primer autor. 


\section{Introducción}

En este trabajo se muestra el modelo matemático obtenido para una estructura de control PID de ganancia variable implementada mediante amplificadores operacionales. Para lograr un comportamiento no lineal en el controlador, se añadió un transistor JFET de canal $\mathrm{N}$ en la entrada del amplificador operacional que ejecuta un algoritmo de control PID convencional. En este caso, se requiere controlar la temperatura interior de un horno eléctrico que calienta mediante 2 resistencias eléctricas conectadas en serie. Este sistema tiene una alta constante de tiempo térmica. El horno funciona a 127 Voltios, y consume 6 Amperios. Se ha estado trabajando realizando este tipo de modificaciones a los algoritmos de control convencionales con el objetivo de conseguir un control más rápido y preciso de la temperatura, para aplicaciones de tratamiento térmico de muestras metalográficas.

El controlador PID modificado que se considera en este trabajo, está diseñado para aumentar su ganancia conforme el punto de consigna se ajusta a un valor más grande. El Control PID No Lineal propuesto ha sido probado experimentalmente con éxito. Se conservan las ventajas de un control PID, con la novedad de que la ganancia del controlador será mayor cuando el punto de referencia se incremente, reforzando el nivel del voltaje de control (Vcon) haciendo que el punto de referencia de la variable se alcance más rápidamente que con la versión convencional, y manteniéndose en el valor deseado.

\section{Metodología}

La estructura de control PID implementada mediante amplificadores operacionales mostrada en la Figura 1 es la originalmente propuesta por K. Ogata en [1]. El autor explica que el segundo AO funciona como inversor de signo y como ajustador de ganancia.

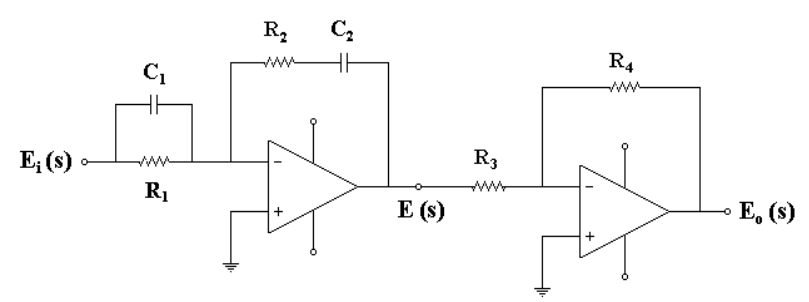

Figura 1 Estructura PID propuesta por K. Ogata. [1]
Tomando como referencia el circuito de la Figura 1, La función de transferencia que resulta de su análisis es la ecuación (1).

$\frac{E_{o}(s)}{E_{i}(s)}=\frac{R_{4}\left(R_{1} C_{1}+R_{2} C_{2}\right)}{R_{3} R_{1} C_{2}}\left[1+\frac{1}{\left(R_{1} C_{1}+R_{2} C_{2}\right) s}+\frac{R_{1} C_{1} R_{2} C_{2}}{R_{1} C_{1}+R_{2} C_{2}} s\right]$

La ecuación (2) es el esquema al que corresponde esta estructura, O’Dywer lo llama: "controlador PID ideal" [2]. Cuando el controlador se expresa con la función de transferencia canónica de la ecuación (2), Kp se denomina ganancia proporcional, Ti tiempo integral y Td tiempo derivativo [1].

$$
\frac{E_{o}(s)}{E_{i}(s)}=K_{p}\left(1+\frac{T_{i}}{s}+T_{d} s\right)
$$

A partir de la ecuación (1), se obtiene que la ganancia proporcional $\mathrm{Kp}$, el tiempo integral Ti y el tiempo derivativo $\mathrm{Td}$ son:

$$
\begin{aligned}
& \mathbf{K}_{\mathrm{p}}=\frac{\mathbf{R}_{4}\left(\mathbf{R}_{1} \mathbf{C}_{1}+\mathbf{R}_{2} C_{2}\right)}{\mathbf{R}_{3} \mathbf{R}_{1} C_{2}} \\
& \boldsymbol{T}_{\mathrm{i}}=\frac{1}{\mathbf{R}_{1} C_{1}+\mathbf{R}_{2} C_{2}} \\
& \mathbf{T}_{\mathrm{d}}=\frac{\mathbf{R}_{1} C_{1} \mathbf{R}_{2} C_{2}}{\mathbf{R}_{1} C_{1}+\mathbf{R}_{2} C_{2}}
\end{aligned}
$$

El esquema de control PID ideal, también puede expresarse mediante la función de transferencia canónica mostrada en (6), en este caso, $\mathrm{Kp}$ se denomina ganancia proporcional, $\mathrm{Ki}$ ganancia integral y Kd ganancia derivativa [1].

$$
\frac{E_{o}(s)}{E_{i}(s)}=K_{p}+\frac{K_{i}}{s}+K_{d} s
$$

Para éste controlador:

$$
\begin{aligned}
& K_{\mathbf{P}}=\frac{\mathbf{R}_{4}\left(\mathbf{R}_{1} \mathbf{C}_{1}+\mathbf{R}_{2} \mathbf{C}_{2}\right)}{\mathbf{R}_{3} \mathbf{R}_{1} \mathbf{C}_{2}} \\
& \mathbf{K}_{\mathrm{i}}=\frac{\mathbf{R}{ }_{4}}{\mathbf{R}_{3} \mathbf{R}_{1} \mathbf{C}_{2}} \\
& \mathbf{K}_{\mathrm{d}}=\frac{\mathbf{R}_{4} \mathbf{R}_{2} C_{1}}{\mathbf{R}_{3}}
\end{aligned}
$$

Para obtener las ganancias de la estructura originalmente propuesta por K. Ogata se utilizan los valores comerciales determinados en un trabajo previo [3]. El circuito se muestra en la Figura 2. Las ganancias resultan:

$K_{p}=56.21$
$K_{i}=21276.59$
$K_{d}=5.6 \times 10^{-4}$ según (7) según (8) según (9) 

escribe:

Sustituyendo estos valores en (6) se

$\frac{E_{o}(s)}{E_{i}(s)}=56.21+\frac{21276.59}{s}+0.00056 \cdot s$

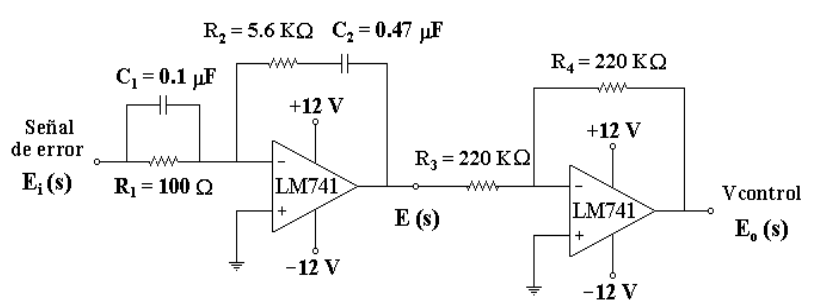

Figura 2 Valores determinados luego de una sintonía fina en el sitio para la estructura PID propuesta por K. Ogata. [3].

\section{Desarrollo}

El circuito PID Normal propuesto por Ogata, se modifica para hacerlo PID de Ganancia Variable dependiendo del punto de referencia, con el lazo de retroalimentación ajustado en la salida del controlador PID convencional. Para ello, se añade un Transistor JFET como se muestra en la Figura 3.

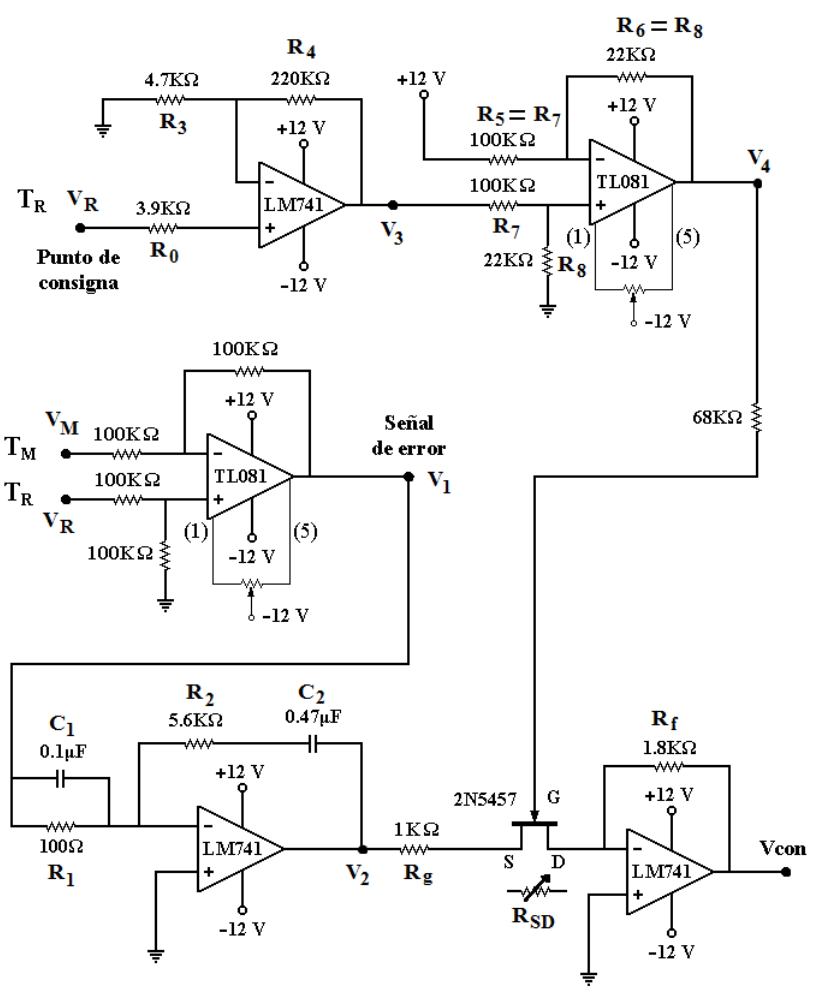

Figura 3 Estructura PID No Lineal obtenida al agregar un Transistor JFET

La idea consiste en adecuar la señal electrónica que representa el punto de consigna para excitar la terminal Gate del transistor JFET $\mathrm{y}$ hacerlo funcionar como una resistencia variable de Source-Drain (RSD).
El valor de RSD dependerá de la magnitud al cual se ajuste el punto de referencia deseado y actúe para ajustar la ganancia del controlador PID y hacer que su comportamiento sea no lineal, similar a lo presentado en [4].

Éste voltaje primero se amplifica en el AO configurado como amplificador no inversor multiplicándolo por 46.8, luego en el AO configurado como restador se realizan dos operaciones simultáneamente, primero se resta de -12 volts y luego se divide entre 4.54 . De esta forma, dependiendo del valor del voltaje de referencia el voltaje de salida del AO configurado como restador tendrá un valor de entre 0 a 0.7 voltios positivo con lo cual el transistor JFET se comportará como resistencia variable, aproximadamente de $100 \Omega$ a $1000 \Omega$.

Continuando con la explicación de la Figura 3, el voltaje que representa la señal de error se ingresa al Amplificador operacional donde se configuró el control PID normal; Luego en la etapa de inversión de signo, la resistencia $\mathrm{Rg}$ se conecta en serie a la terminal Source del Transistor JFET, con lo cual la resistencia interna RSD del 2N5457, se suma en serie con la resistencia $\mathrm{Rg}$. Lo anterior, en conjunto con el valor de Rf es lo que hará que la ganancia del controlador varíe en función de la magnitud del valor de referencia seleccionado.

Los valores comerciales mostrados en la Figura 3, se obtuvieron experimentalmente, consiguiendo un buen desempeño en la aplicación requerida.

Las ecuaciones que describen el control PID No Lineal mostrado en la Figura 3, se establecen a partir del método de las impedancias descrito por K. Ogata en [1].

Definiendo:

$V_{1}=V_{R}-V_{m}$

$V_{2}=-\frac{R_{2}}{R_{1}}\left[\frac{\left(R_{1} C_{1} S+1\right)\left(R_{2} C_{2} S+1\right.}{R_{2} C_{2} S}\right] V_{1}$

Simplificando

$V_{2}=-\left(R_{2} C_{1} S+\frac{1}{R_{1} C_{2} S}+\frac{R_{1} C_{1}+R_{2} C_{2}}{R_{1} C_{2}}\right) V_{1}$

Sustituyendo $V_{1}$ en $V_{2}$ se tiene:

$V_{2}=-\left(R_{2} C_{1} S+\frac{1}{R_{1} C_{2} S}+\frac{R_{1} C_{1}+R_{2} C_{2}}{R_{1} C_{2}}\right)\left(V_{R}-V_{m}\right)$

GARCÍA-CORTÉS, Julio Zenón, CARRILLO-GARCÍA, Francisco Javier, TAMAYO-LOEZA, Erick del Jesus y MAY-CEN, Ivan de Jesus. Modelado Matemático de un Controlador PID de Ganancia Variable dependiente del Punto de Consigna en la Salida de un PID Convencional. Revista de Operaciones Tecnológicas. 2019 
Análisis del punto de consigna

$$
\begin{aligned}
& V_{3}=\left(1+\frac{R_{3}}{R_{4}}\right) V_{R} \\
& V_{4}=\frac{R_{6}}{R_{5}}\left(V_{3}-12\right)
\end{aligned}
$$

Sustituyendo $V_{3}$ en $V_{4}$ se tiene:

$$
\begin{aligned}
& V_{4}=\frac{R_{6}}{R_{5}}\left[\left(1+\frac{R_{3}}{R_{4}}\right) V_{R}-12\right] \\
& V_{G}=V_{4} \text { Por lo tanto } \\
& V_{G}=\frac{R_{6}}{R_{5}}\left[\left(1+\frac{R_{3}}{R_{4}}\right) V_{R}-12\right]
\end{aligned}
$$

\section{Analizando el JFET}

$$
\begin{aligned}
& \frac{V_{\text {control }}}{V_{2}}=-\left[\frac{R_{f}}{R_{g}+R_{S D}}\right] \\
& V_{\text {control }}=-\left[\frac{R_{f}}{R_{g}+R_{S D}}\right] V_{2}
\end{aligned}
$$

Sustituyendo $V_{2}$ en $V_{\text {control }}$ se obtiene:

$$
\begin{gathered}
V_{\text {control }}=-\frac{R_{f}}{\left(R_{g}+R_{S D}\right)}\left[-\left(R_{2} C_{1} S+\frac{1}{R_{1} C_{2} S}+\frac{R_{1} C_{1}+R_{2} C_{2}}{R_{1} C_{2}}\right)\left(V_{R}\right.\right. \\
\left.\left.-V_{m}\right)\right]\left(V_{R}-V_{m}\right)
\end{gathered}
$$

Simplificando y debido al efecto del inversor de signo resulta:

$V_{\text {control }}=\frac{R_{f}}{\left(R_{g}+R_{S D}\right)}\left[\left(R_{2} C_{1} S+\frac{1}{R_{1} C_{2} S}+\frac{R_{1} C_{1}+R_{2} C_{2}}{R_{1} C_{2}}\right)\right]\left(V_{R}-V_{m}\right)$

\section{Resultados}

Con el propósito de comprobar la validez del modelo matemático, se decidió comparar los análisis de estabilidad del modelo estudiado por K. Ogata en [1] contra la modificación propuesta en este trabajo.

Primero se obtienen los gráficos para el modelo indicado por K. Ogata. Para trabajar con Matlab (10) se escribe:

$$
\frac{E_{o}(s)}{E_{i}(s)}=\frac{0.00056 \cdot s^{2}+56.21 \cdot s+21276.59}{s}
$$

La función obtenida del modelo es impropia, por ello se decidió determinar la estabilidad mediante la obtención y análisis de los diagramas de Bode.
En la Figura 4, se muestran las trazas de Bode de (19) obtenidas con ayuda de MatLab, su análisis indica que la estructura es estable, puesto que la curva de fase ni siquiera cruza los -180 grados.

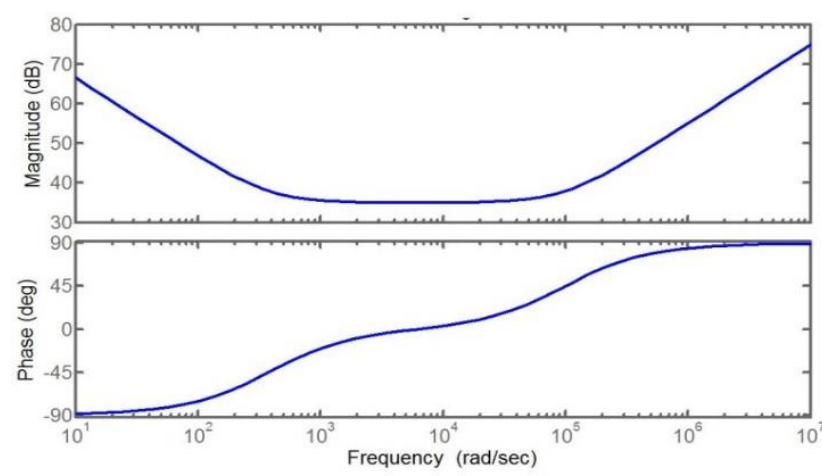

Figura 4 Trazas de Bode para la estructura original de K. Ogata

Por otra parte, para la estructura PID de ganancia variable propuesta, se considera un momento intermedio del proceso de control. Lo anterior dado que la ganancia RSD es variable. En la Tabla I se muestra una correspondencia de los valores que adquiere RSD conforme el punto de consigna cambia. Nótese que conforme el valor de referencia se incrementa, el valor de RSD disminuye, esto ocasionará que a mayores puntos de consigna, la ganancia del controlador sea más grande. Para calcular RSD se realizaron mediciones en sitio y luego se aplicó la fórmula:

$$
\mathrm{R}_{\mathrm{SD}}=\frac{\mathrm{V}_{\mathrm{SD}}}{\mathrm{I}_{\mathrm{SD}}}
$$

\begin{tabular}{|r|r|}
\hline $\mathrm{T}_{\mathrm{R}}(\mathrm{V})$ & $\mathrm{R}_{\mathrm{SD}}(\Omega)$ \\
\hline 0.021 & 875 \\
\hline 0.0405 & 785.7143 \\
\hline 0.05 & 744.186 \\
\hline 0.0758 & 645.3382 \\
\hline 0.101 & 567.9443 \\
\hline 0.126 & 500 \\
\hline 0.1503 & 451.6129 \\
\hline 0.1756 & 417.3228 \\
\hline 0.2 & 406.25 \\
\hline 0.2508 & 395.3488 \\
\hline
\end{tabular}

Tabla I Valores de $\mathrm{R}_{\mathrm{SD}}$ en diferentes puntos de referencia

Para obtener la función de transferencia basta reconocer que $\left(V_{R}-V_{m}\right)$ es el voltaje que representa la señal de error, y por lo tanto es la señal de voltaje que en este caso se considera como la entrada. Ahora bien, si el punto de referencia se establece en $200^{\circ}$; según la Tabla I, se puede suponer un valor para RSD de 406.25 $\Omega$. Sustituyendo éste y los valores mostrados en la Figura 3, en (18), se puede escribir:

GARCÍA-CORTÉS, Julio Zenón, CARRILLO-GARCÍA, Francisco Javier, TAMAYO-LOEZA, Erick del Jesus y MAY-CEN, Ivan de Jesus. Modelado Matemático de un Controlador PID de Ganancia Variable dependiente del Punto de Consigna en la Salida de un PID Convencional. Revista de Operaciones Tecnológicas. 2019 


$$
\frac{\mathrm{V}_{\text {control }}}{\left(\mathrm{V}_{\mathrm{R}}-\mathrm{V}_{\mathrm{m}}\right)}=1.28\left(0.00056 \mathrm{~S}+\frac{21,276.5957}{\mathrm{~S}}+56.21\right)
$$

Simplificando en MatLab se obtiene:

$\frac{\mathrm{V}_{\text {control }}}{\left(\mathrm{V}_{\mathrm{R}}-\mathrm{V}_{\mathrm{m}}\right)}=\frac{0.00001491 \mathrm{~S}^{2}+1.497 \mathrm{~S}+566.5}{\mathrm{~S}}$

Cabe destacar que por cuestiones de espacio los coeficientes de (21) se han redondeado, sin embargo, en los cálculos en Matlab si se consideraron las cantidades completas.

En la Figura 5 se muestra el diagrama de Bode para el caso considerado. Analizando el gráfico se observa que el sistema es estable, puesto que la curva de fase ni siquiera cruza el eje de -180 grados.

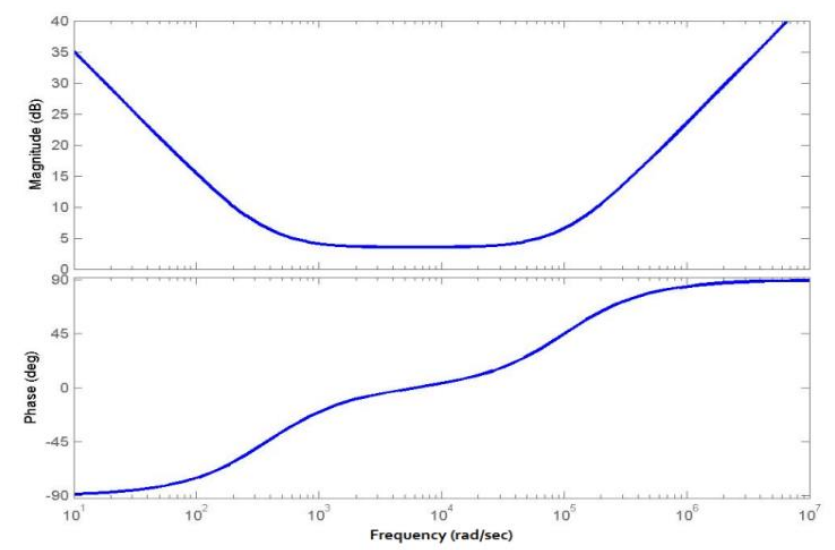

Figura 5 Trazas de Bode para la estructura PID No Lineal de (21)

Por otra parte, en la Figura 6 se muestran los resultados de un caso de prueba con el valor de referencia ajustado a $200^{\circ} \mathrm{C}$. Se incluye la curva de la respuesta que tendría un controlador ideal, lo cual se obtuvo realizando un modelado empírico del sistema de control en sitio; este modelo ideal está ajustado para alcanzar el punto de consigna en un tiempo muy cercano al que lo haría en lazo abierto, lo cual sería muy difícil de lograr sin tener un sobreimpulso considerable, pero proporciona una idea de que tan eficientes son los controladores reales bajo estudio. Entonces, comparando el comportamiento del controlador PID convencional con el comportamiento del controlador PID de ganancia variable propuesto, se comprueba que existe una ligera mejora en el funcionamiento deseado.

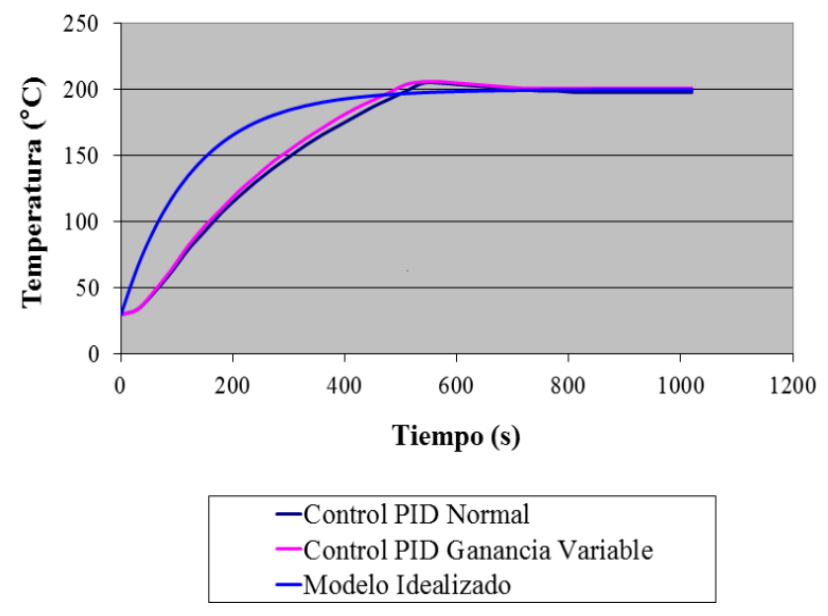

Figura 6 Grafica de resultados de pruebas reales a $200^{\circ} \mathrm{C}$

\section{Conclusiones}

Se obtuvo un modelo matemático para un controlador PID con ajuste de ganancia implementado con Amplificadores Operacionales.

El modelo brinda una aproximación del comportamiento real del circuito propuesto y hace posible efectuar análisis de estabilidad, mediante los cuales se determinó que la estructura implementada es estable.

El modelo servirá de base para futuros trabajos, donde se pretende utilizar la estructura para controlar sistemas SISO que tengan tiempos de retardo grandes o que presenten un comportamiento no lineal.

\section{Referencias}

[1] K. Ogata, "Ingeniería de Control Moderna," Ed. Pearson Educación S.A., $4^{\mathrm{a}}$ ed., España, 2003.

[2] A. O'Dwyer, "HandBook of PI and PID Controller Tunning Rules", Ed. Imperial College Press, 2a ed., Irlanda, 2006.

[3] J. Z. García, "Ensayo experimental sobre 2 estructuras de control PID proyectadas mediante amplificadores operacionales, en una aplicación de control de temperatura," en memorias de $5^{\circ}$ Congreso Internacional de Ingeniería Electromecánica y de Sistemas, pp. 262-267, México, D.F., 2008, ISBN: 978-607-414-049-1 
[4] J. Z. García, A. Rodríguez, and J. H. Caltenco, "Controlador ProporcionalIntegral No Lineal Aplicado a un Sistema de Alta Inercia Térmica, Utilizando el Método de Control por Paquetes con base de Tiempo Variable". en memorias de XII Congreso Nacional de Ingeniería Electromecánica y de Sistemas, pp. 253-258, México, D.F., 2010. Rashid, M., Electrónica de Potencia, Circuitos, Dispositivos y Aplicaciones, Ed. Pearson Educación de México, S. A. De C.V., $3^{\text {a }}$ ed., México, pp. 500-502, 1994.

[5] Rashid, M., Electrónica de Potencia, Circuitos, Dispositivos y Aplicaciones, Ed. Pearson Educación de México, S. A. De C.V., $3^{\text {a }}$ ed., México, pp. 500-502, 1994.

[6] Dorf, R. C. y Bishop, R. H., Sistemas de Control Moderno, Ed. Pearson Educación S. A., 10ª ed., España, pp 696, 2005. 